\title{
Linear Synchronous Motor Model
}

\author{
Ilya Syrkin $^{1^{*}}$ \\ ${ }^{1}$ T.F. Gorbachev Kuzbass State Technical University, 650000 Vesennya Street, 28, Kemerovo, the \\ Russian Federation
}

\begin{abstract}
Liner synchronous motor (LSM) is perspective motor for milling machines and other manufacturing equipment such as $3 \mathrm{D}$ printers, laser cutting and engraving machines, etc. Some different construction of LSM can be found. The LSM with permanent magnets is modeled in this paper. Comsol Multiphysics is used for finite element model of LSM. Motor stator is built using rear earth magnets N52, an anchor consists of 6 teeth with 3-phase winding. Large cogging force is the problem of LSM, so there is task to reduce this force. This task can be solved by motor geometry optimization. Geometric parameter of motor is represented by variables. It allowed using optimization methods for best geometry search. FEM model with two different mesh sizes is analysed in this paper. Each mesh allows find solution but calculation time and tolerance are different. During experiments, optimal size of tooth for maximal driving force is found. Cogging force is also reduced.
\end{abstract}

\section{Introduction}

In modern engineering, it is necessary to intensify the production processes of various parts. One of the methods is high-speed milling. The creation of high-speed milling machines requires the creation of high-speed drives with high dynamic performance. Used in most cases, drives based on synchronous and asynchronous motors with mechanical transmission "spindle screw" cannot provide high speeds and accelerations of movement of the machine tool. To avoid this problem a drive can be built using linear electric motors (LM). LM have high dynamic characteristics. This will determine their widespread use in high-speed and accurate drive systems. The main advantage of drives based on the LM base compared to drives based on conventional motors is the absence of a mechanism that converts the rotation of the motor shaft to linear movement of the working element.

There are several basic designs of LM, among which the most widespread engines with permanent magnets. Any LM is part of a drive that must be designed to obtain the required dynamic performance. Drive development cannot be done without creating a LM model. Modeling LM can be done in several ways, for example, to build a simplified model based on simple physical equations of electromagnetic induction. [1]. Simplified models do not allow taking into account additional forces affecting the dynamics of LD. These forces include the so-called cogging force. Many scientists are engaged in the elimination of its influence. [2-9, 11-15]. In this paper, it is proposed to construct a finite element model of

\footnotetext{
*Corresponding author: syrkin@kuzstu.ru
} 
LM in order to take into account the influence of all geometric and electrical parameters of the engine on its dynamic characteristics.

\section{Linear motor FEM model development}

COMSOL Multiphysics is a finite element analysis program (Finite Element Analysis, FEM) that allows you to solve a wide range of problems in the simulation of physical phenomena. Among the possibilities, there is a simulation of electromagnetic fields in the AC/DC module.

The linear motor consists of a stator with permanent magnets and an anchor with coils. In fig. 1 . The stator is represented by a metal substrate 1 and magnets $3-12$, an anchor by a core 21 and windings 22-33.

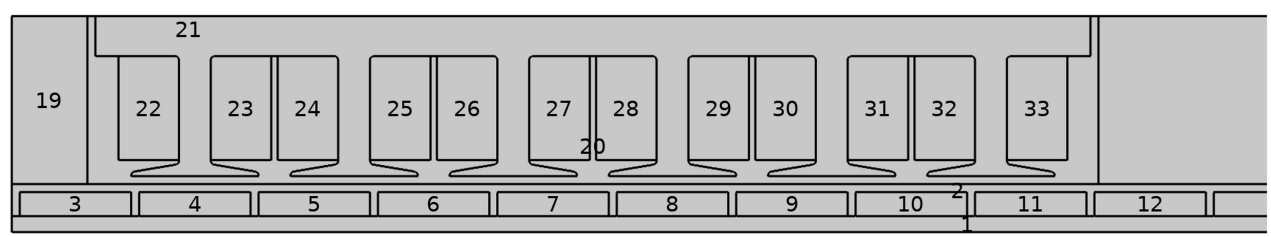

Fig. 1. Linear motor schematic

In order to expand the possibilities of modeling, all dimensions are given through parameters, which allows us to construct many variants of engine design. The main parameters of the motor are represented in table 1.

Table 1. Linear motor geometry parameters

\begin{tabular}{|l|l|}
\hline Parameter name & Value \\
\hline Anchor length & $125 \mathrm{~mm}$ \\
\hline Motor width & $26 \mathrm{~mm}$ \\
\hline Tooth count & 6 \\
\hline Coil count & 3 \\
\hline Magnet width & $14 \mathrm{~mm}$ \\
\hline Magnet height & $3 \mathrm{~mm}$ \\
\hline MAgnet step & $15 \mathrm{~mm}$ \\
\hline Current density & $10 \mathrm{~A} / \mathrm{m}^{2}$ \\
\hline
\end{tabular}

The selected motor dimensions allow the creation of a 3-phase LM similar in magnetics to a 3-phase brushless motor with 6 teeth and 8 magnets. In this case, according to the calculator [10], the windings are wound according to the $\mathrm{ABCABC}$ scheme, i.e. phases $\mathrm{A}$, $\mathrm{B}, \mathrm{C}$ alternate in sequence, the winding direction is the same. The current density of $10 \mathrm{~A} / \mathrm{m}^{2}$ is selected based on the air-cooling of the winding. If you change the cooling method, it is acceptable to increase the current density. Magnets selected rare earth, alloy N52. The magnet model uses the standard Nonlinear Permanent Magnet material, in which the magnetization curve is set in accordance with the magnetic properties of the selected magnet.

Engine simulation is performed using a finite element analysis; therefore, the geometric model must be converted to a grid for transfer to the calculation module. Two grids are used in the work: coarse (Fig. 2a) and fine (Fig. 2b). The size of the grid affects the accuracy of calculations and the time of calculations. 


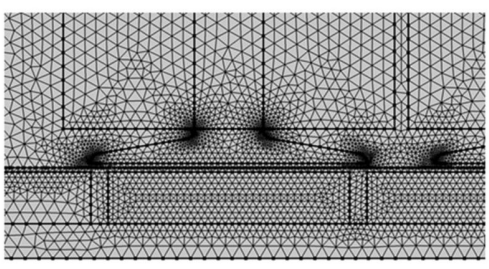

a)

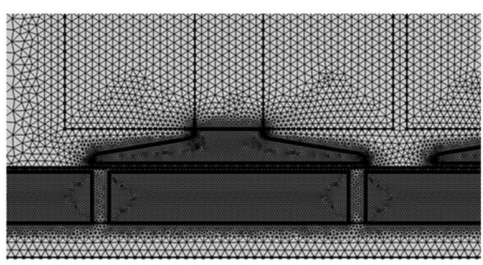

b)

Fig. 2. FEM meshes

The construction of the force dependence on the position of the armature relative to the stator can be done by adding the "Moving Mesh" physics to the model. This will allow you to change the position of the anchor depending on the time. Since the pitch of the magnets is $15 \mathrm{~mm}$, and the direction of the magnetic field of the magnets alternates, it is enough to move the anchor by $30 \mathrm{~mm}$.

To build a graph of cogging force, you can do by removing the current in the windings. Large cogging force must be reduced to achieve uniformity of movement of the armature of the engine. This is possible only by optimizing the geometry of the engine.

To determine the effect of the geometry of the electric motor on the magnitude of the force, experiments were performed: one of the parameters varied and the forces were built with and without electric current flowing. Conducted one experiment to change the height of the base of the tooth at its outer part (Fig)

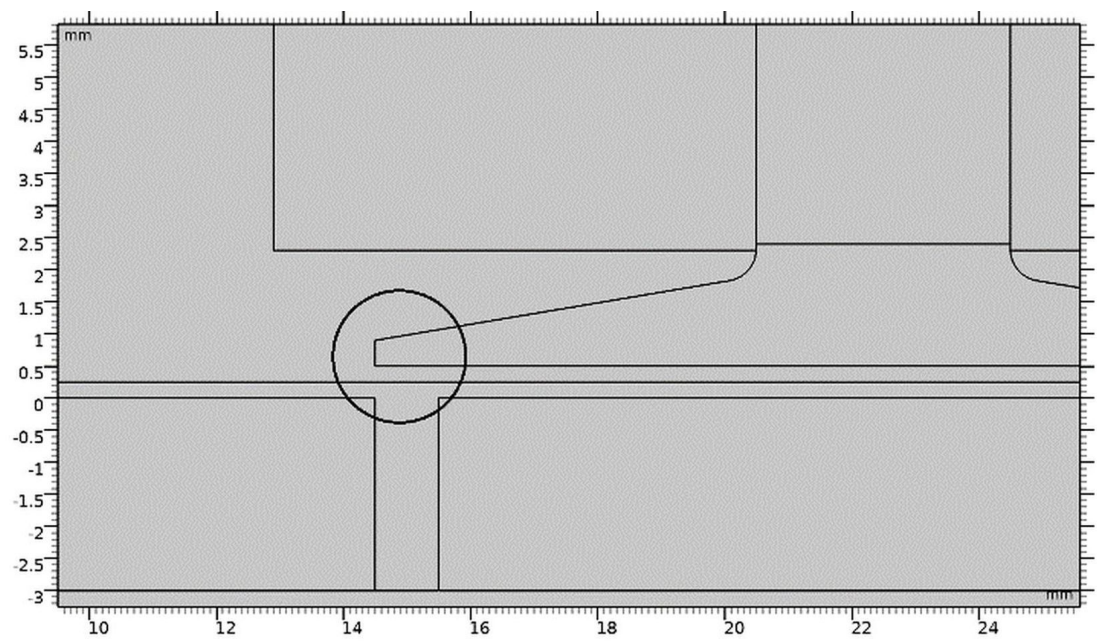

Fig. 3. Tooth height

The dynamic characteristics of the engine are completely determined by its geometry. As proof of this statement, the results of studies of the maximum developed force depending on the values of the geometric parameters of the engine will be presented.

\section{Results}

Magnetic fields modelling shows us flux distribution depicted on fig 4 . 


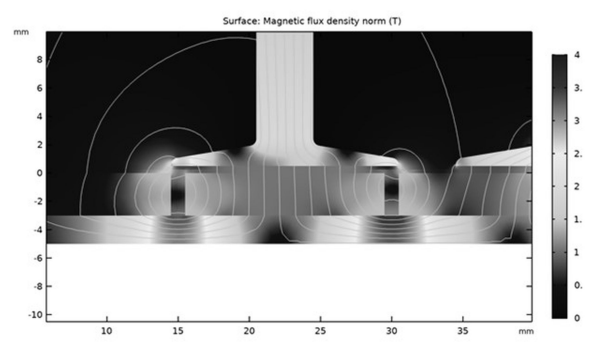

a)

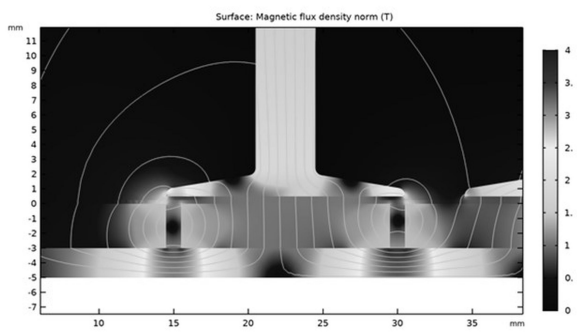

b)

Fig. 4. Magnetic flux

The plotting of the force dependence on the position of the anchor was carried out at two different meshes.

The calculation time of the coarse mesh was 190 seconds; for the fine mesh, 491s. The results are shown in Fig. 5.

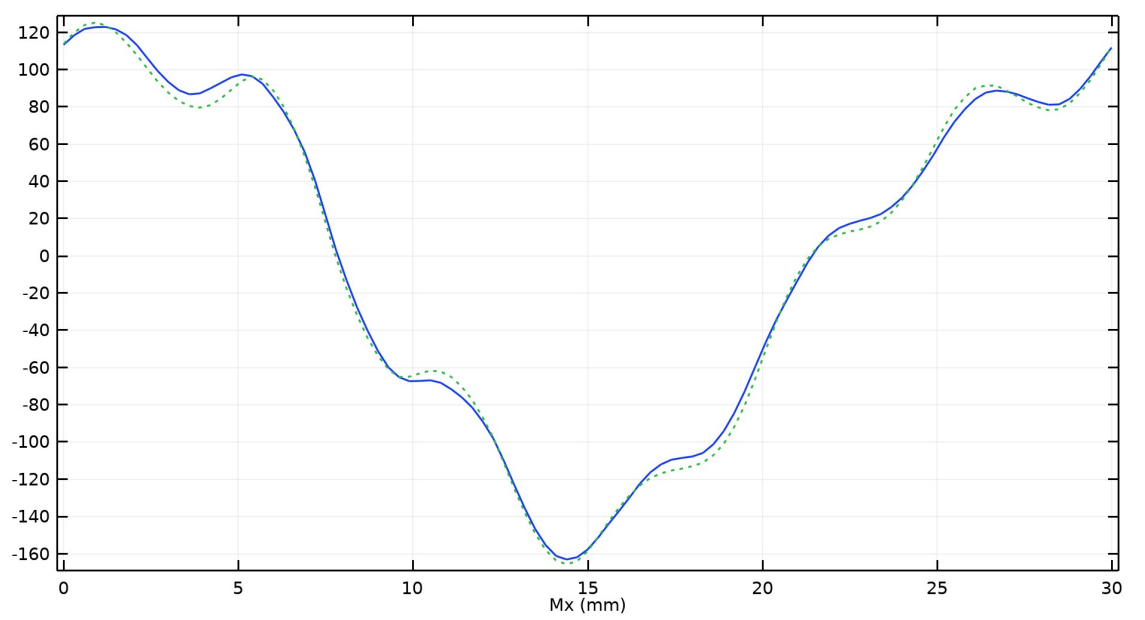

Fig. 5. Magnetic force in motor. (Solid line for coarse mesh, dotted is for fine mesh). Cogging force depicted in Fig 6

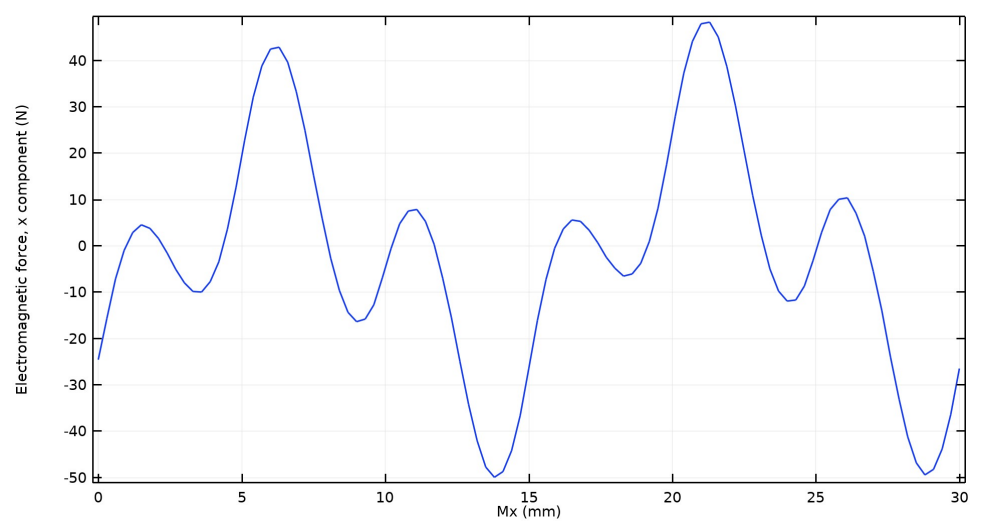

Fig. 6. Cogging force. 
As it can be seen from the graph, when moving the armature of the engine, even without flowing current, the magnitude of the force reaches $50 \mathrm{~N}$. This value is large, measures are needed to reduce it.

As a result of studying the effect of tooth geometry on the magnitude of the effort, the following data were obtained:

Table 2. Modelling results

\begin{tabular}{|l|l|l|}
\hline Tooth height, $\mathrm{mm}$ & Cogging force, $\mathrm{N}$ & Working force, $\mathrm{H}$ \\
\hline 0.2 & 44.7 & 106 \\
\hline 0.4 & 50.1 & 105 \\
\hline
\end{tabular}

The results in the table show that the geometry of the tooth affects the amount of effort. The effect of different geometrical dimensions of the engine also affects the force developed. The results of these studies are shown in Fig 7.

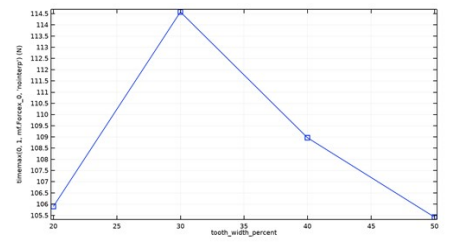

a) Relative tooth width

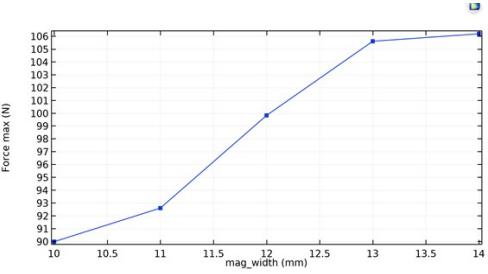

b) Magnet width

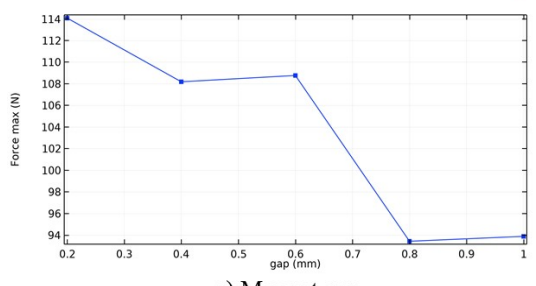

c) Magnet gap

Fig. 7. Modelling results

\section{Discussion}

The simulation result showed that the finite element method allows to create a linear motor model. The size of the grid significantly affects the computation time, but the difference between the values of the efforts diverge by no more than $5 \%$. Consequently, the use of a fine mesh is necessary for the final calculations, and a coarse one for carrying out a multitude of experiments to study the effect of various parameters of LM on the force developed.

Experiments on changing the geometric parameters of the LM showed that the force developed and the tooth force depend on the size of the teeth, the distance between the armature and the stator of the engine. This suggests that with certain combinations of sizes, it will be possible to achieve a minimum barb force with a maximum developed thrust.

\section{Conclusion}

The paper presents a linear motor model, showing the possibility of determining the optimal geometric dimensions to achieve maximum dynamic performance. 


\section{References}

1. Zhang, Dailin \& Kong, Ching \& P Chen, Y. Proceedings of the 17th World Congress The International Federation of Automatic Control Seoul, Korea, 6 (2008)

2. Hai-Hua Mua,b, , Yun-Fei Zhou, Xin Wena, Yan-Hong Zhou, Mechatronics 19, 577 (2009)

3. Nariman Roshandel Tavana, Abbas Shoulaie, Energy Conversion and Management 52, $349(2011)$

4. H.M. Hasanien, Energy Convers Manage, 51:98 (2010)

5. Raminosoa T, Farooq JA, Djerdir A, Miraoui A. Energy Convers Manage, 50:1356 (2009)

6. Li T, Slemon G. IEEE Trans Magn, 24:11 (1988)

7. Cruise RJ, Landy CF. IEEE Africon, 623 (1999)

8. I Takeo, G. Slemon, A IEEE Trans Magn, 29:2, 2028 (1993)

9. Zhu ZQ, Xia ZP, Howe D, Mellor PH. IEE Proc - Elect Pow Appl;144(4):277 (1997)

10. Li T, Slemon, IEEE Trans Magn, 24(11):2901 (1988)

11. Yoshimura T, Kim HJ, Watada M, Torii S, Ebihara D. IEEE Trans Magn, 31(6):3728, 30 (1995)

12. Shen, Jian-Xin, et al. The International Journal for Computation and Mathematics in Electrical and Electronic Engineering, 35:2, 641 (2016)

13. Islam, Rakib, et al. Industry Applications, IEEE Transactions on, 45:1, 152 (2009)

14. Zhu, Xiaofeng, et al. IEEE Transactions on Magnetics, 53:6, 1 (2017) 